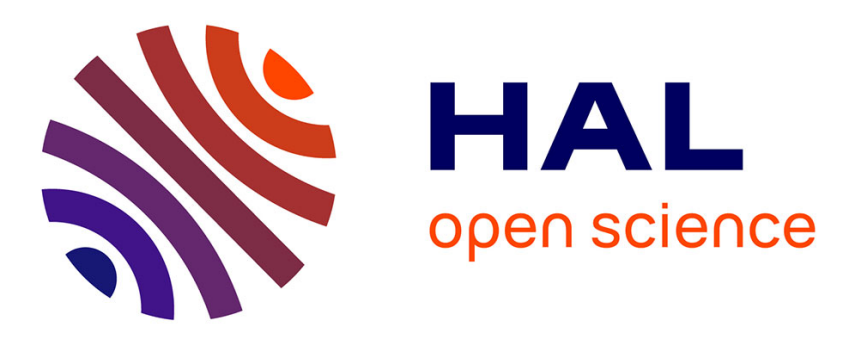

\title{
Mass-Selected FeCo Clusters Embedded in a Carbon Matrix as Benchmark Nanocatalysts
}

Véronique Dupuis, Ghassan Khadra, Juan Martín Montejano-Carrizales, Florent Tournus, Faustino Aguilera-Granja, Alexandre Tamion

\section{- To cite this version:}

Véronique Dupuis, Ghassan Khadra, Juan Martín Montejano-Carrizales, Florent Tournus, Faustino Aguilera-Granja, et al.. Mass-Selected FeCo Clusters Embedded in a Carbon Matrix as Benchmark Nanocatalysts. ACS Applied Nano Materials, 2019, 2 (5), pp.2864-2872. 10.1021/acsanm.9b00313 . hal-02126950

\section{HAL Id: hal-02126950 \\ https://hal.science/hal-02126950}

Submitted on 13 May 2019

HAL is a multi-disciplinary open access archive for the deposit and dissemination of scientific research documents, whether they are published or not. The documents may come from teaching and research institutions in France or abroad, or from public or private research centers.
L'archive ouverte pluridisciplinaire HAL, est destinée au dépôt et à la diffusion de documents scientifiques de niveau recherche, publiés ou non, émanant des établissements d'enseignement et de recherche français ou étrangers, des laboratoires publics ou privés. 


\title{
Mass-Selected FeCo Clusters Embedded in a Carbon Matrix as Benchmark Nanocatalysts
}

\author{
Veronique Dupuis, ${ }^{*}{ }^{\dagger}$ (i) Ghassan Khadra, ${ }^{\dagger}$ Juan Martín Montejano-Carrizales, ${ }^{\ddagger}$ Florent Tournus, ${ }^{\dagger}$ (1) \\ Faustino Aguilera-Granja, ${ }^{\ddagger} \S_{\odot}$ and Alexandre Tamion ${ }^{\dagger}$ \\ ${ }^{\dagger}$ Institut Lumière Matière, UMR 5306, Université Lyon 1-CNRS, Université de Lyon, 69622 Villeurbanne, France \\ "Instituto de Fisica "Manuel Sandoval Vallarta”, Universidad Autónoma de San Luis Potosí, 78000 San Luis Potosí, Mexico \\ ${ }^{\S}$ Centro de Física de Materiales CFM-MPC CSIC-UPV/EHU, Donostia International Physics Center (DIPC), 20018 San Sebastián, \\ Spain
}

Supporting Information

ABSTRACT: Despite cobalt and iron-carbon interactions playing an important role in environmental chemistry activities, major questions are still unresolved on the prevailing mechanism, surface state, required structure, and the origin of apparent particle size dependence in carbon nanotube synthesis or other heterogeneous catalytic process such as Fischer-Tropsch (F-T) synthesis. In this paper, we focus our attention on well-defined bimetallic FeCo clusters embedded in a carbon matrix to study separately size, alloy, and interface effects on physical properties. First the size-dependent evolution of the intrinsic structural and magnetic properties was estimated by using density-functional ab initio calculations for relaxed B2 (CsCl-like) FeCo nanoclusters. Then, assemblies of mass-selected clusters preformed in the gas phase by using the low-energy cluster beam deposition (LECBD) technique and coated with a carbon

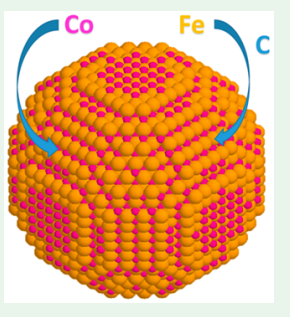
film were investigated by use of various experimental techniques that include transmission electron microscopy (TEM) and superconducting quantum interference device (SQUID) magnetometry as well as synchrotron techniques such as extended Xray absorption fine structure (EXAFS) and X-ray magnetic circular dichroism (XMCD). All these results show that the carbon solubility is a size-dependent process and that annealing allows us to reach the chemically ordered B2 phase only for FeCo nanoparticles larger than $5 \mathrm{~nm}$ in diameter.

KEYWORDS: size effect, bimetallic nanoalloy, chemical order, superparamagnetism, carbide formation and chemical reactivity

\section{INTRODUCTION}

Being able to take full advantage of synergistic effects of bimetallic nanoalloys is a real challenge as well as providing solutions for the substitution of critical metals (rare earth or platinum group metals). ${ }^{1}$ Like iron, the most abundant and low cost metal, cobalt is commonly combined with other metals to create alloys. Cobalt and iron-carbon interactions play an important role in various industrial activities such as carbon nanotube synthesis and liquid fuel production by the Fischer-Tropsch $(\mathrm{F}-\mathrm{T})$ process with an increasing interest for FeCo catalysts. ${ }^{2,3}$ Nevertheless, among other factors, the ongoing challenge remains the influence of the catalyst particle size (the size effect) and the incorporation of an additional metal (the alloying effect) in the surface-specific activity (turnover frequency, TOF). ${ }^{4,5}$

Moreover, pure $\mathrm{Fe}$ and $\mathrm{Co}$ are both ferromagnetic with different bulk structures, bcc and hcp, respectively. Despite having the largest saturation magnetization at ambient temperature, the bulk intermetallic $\mathrm{FeCo}$ compound has a cubic structure stable in the range of $29-70$ at. \% Co. ${ }^{6}$ The B2 (CsCl-type) structure of $\mathrm{FeCo}$ is a chemically ordered structure which can be viewed as two interpenetrating simple cubic sublattices in which the $\mathrm{Fe}$ atoms occupy one sublattice and the Co atoms occupy the other. ${ }^{7}$ Because of this cubic symmetry, $\mathrm{FeCo}$ has a low magnetocrystalline anisotropy and is thus considered as a soft magnet but still having many applications from power generation to microwave communications. ${ }^{8}$

At the nanoscale, crystallized FeCo particles have facets to minimize their surface energy, but both $\mathrm{Fe}$ and Co have almost the same atomic size and similar surface energies. It is thus not obvious to predict the existence of surface segregation in favor of one particular species upon alloying. ${ }^{9}$ In addition, it is very delicate to experimentally confirm the B2 phase in bimetallic nanoparticle assemblies with such a small $Z$ contrast. Furthermore, finite size effects are expected to affect the magnetic properties, which depend on the detailed electronic structure and are consequently related to the chemical reactivity. In particular, the carbon environment may drastically change the magnetic moment and anisotropy of FeCo nanoalloys and could even make this "soft" material appealing for magnetic storage applications. ${ }^{10}$ Indeed, density functional theory calculations recently predicted that tetragonal distortion induced in FeCo alloys doped by carbon could lead to potential new rare-earth-free permanent magnets. ${ }^{11}$

Received: February 18, 2019

Accepted: May 2, 2019

Published: May 2, 2019 


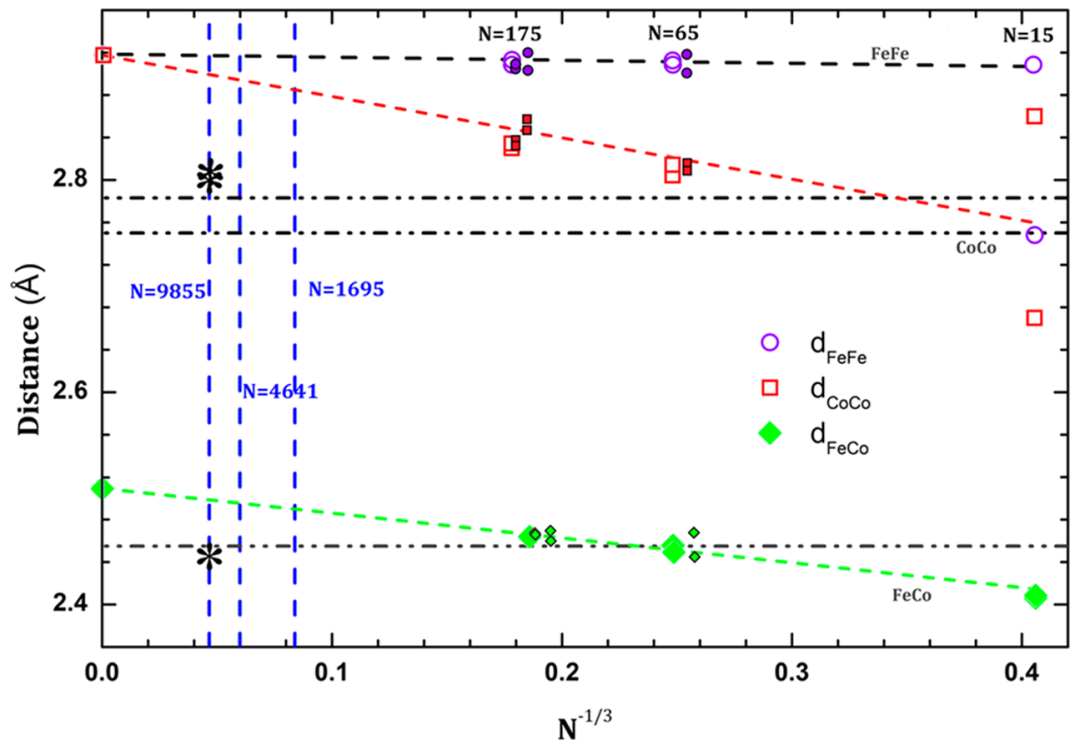

Figure 1. Evolution of the mean interatomic distances of $\mathrm{Fe}-\mathrm{Fe}, \mathrm{Co}-\mathrm{Co}$, and $\mathrm{Fe}-\mathrm{Co}$ calculated for $\mathrm{B} 2 \mathrm{FeCo}$ phase as a function of size $(\mathrm{N}$ is the number of atoms per cluster) from SIESTA calculations (symbols). Vertical lines correspond to clusters with mean diameter 3.7, 4.3, and 6.1 nm, while the horizontal ones correspond to the experimental distances previously obtained on unselected FeCo sample in ref 15 . The large symbols are for rhombic dodecahedron, whereas small symbols correspond to the truncated dodecahedron clusters. The stars in the first blue vertical line correspond to the distances inferred from EXAFS measurements on the largest annealed $(\mathrm{FeCo})_{6 \mathrm{~nm}}$ sample.

In previous papers we have studied the thermally activated chemical order transition in FeCo nanoparticles prepared by low-energy clusters beam deposition (LECBD). ${ }^{10,12}$ Because of the low $Z$ contrast $(\Delta Z=1)$ between Fe and Co atoms, we performed X-ray diffraction experiments on such annealed nanoalloys at the European Synchrotron Radiation Facilities (ESRF) by choosing X-ray energy just below the $\mathrm{Fe}-\mathrm{K}$ absorption edge, where a maximum of anomalous contrast for the atomic scattering factor is expected. Even for the largest FeCo nanoparticles of $5 \mathrm{~nm}$ median diameter, we have not been able to evidence the chemically ordered bulk-like B2 phase by seeing the supplementary (100), (111), and (012) reflections forbidden in the $\mathrm{A} 2$ chemically disordered bcc phase. Such results have been corroborated by calculations of simulated X-ray scattering curves performed for B2 phase FeCo nanoparticles of rhombic dodecahedron shape with equivalent size. ${ }^{12}$ Nuclear magnetic resonance, known as a well-adapted technique for low chemical contrast, has also not been able to evidence B2 chemical order due to the small quantity of matter in our samples. ${ }^{13,14}$ Therefore, a particular strategy based on extended X-ray absorption edge fine structure (EXAFS) experiments and simulations at both $\mathrm{Fe}$ and Co K-edges has been developed as well as accurate magnetic characterizations on as-prepared and annealed FeCo clusters embedded in carbon. ${ }^{15}$ We have shown that upon annealing crystallographic order and magnetic properties are improved, but we have not been able to detect without ambiguity the B2 chemical order probably due to the competition between stable iron carbide formation and cobalt-carbon demixing in bimetallic nanoparticles prepared by clusters deposition.

In this paper, we would like to verify whether carbon solubility effects are size dependent as recently theoretically predicted in phase diagrams for $\mathrm{Ni}-\mathrm{C}$ nanoparticles ${ }^{16}$ and to provide a basic understanding of the true influence of size and alloying effects on the catalytic performance. We have thus prepared benchmark Co- and Fe-based mono- and bimetallic nanoparticles (NPs) by mass-selected LECBD (MS-LECBD), embedded in a carbon matrix, having a size range between 3 and $6 \mathrm{~nm}$ in diameter with a small dispersion. Annealing treatments have been performed to study the structure and magnetism of the equilibrium phase as a function of the $\mathrm{FeCo}$ nanoparticles size by using advanced state-of-the-art characterization techniques. The experimental results are compared to theoretical calculations for $\mathrm{B} 2$ relaxed $\mathrm{FeCo}$ clusters and with C impurities.

\section{DENSITY FUNCTIONAL THEORY (DFT) CALCULATIONS}

First of all, we present density-functional $a b$ initio calculation results performed using the SIESTA code ${ }^{17}$ to study electronic, magnetic, and structural properties of relaxed rhombic dodecahedron FeCo nanoparticles in the B2 phase as a function of the number of atoms per cluster $(N)$. More details on the DFT calculations and the cluster morphology of clusters are described in the Supporting Information where the results of the interatomic distances, the magnetic moments per atom, and the number of holes are reported (Table S1). Figure 1 shows the evolution of the mean interatomic distances ( $\mathrm{Fe}-$ $\mathrm{Fe}, \mathrm{Co}-\mathrm{Co}$, and $\mathrm{Fe}-\mathrm{Co}$ ) for the different sizes in the $\mathrm{B} 2$ phase. The two spots for the same size correspond to the two possible central atom positions (see Figure S1). The large symbols in Figure 1 correspond to rhombic dodecahedron $(N$ $=15,65$, and 175), whereas the small symbols correspond to the truncated dodecahedron clusters $(N=59,145$, and 169). The results plotted in Figure 1 show that the FeCo clusters have a $\mathrm{CoCo}$ interatomic distance shorter than a $\mathrm{FeFe}$ distance with the latter remaining close to that of the bulk for all the sizes. This shows that relaxation effects are larger in the CoCo planes than in the FeFe planes. For both homoatomic distances, it can be noted that as the size of the nanoparticle increases, the calculated values converge (with a $N^{-1 / 3}$ evolution, shown as dashed lines) toward a single bulk distance $(\mathrm{FeFe}=\mathrm{CoCo})$ as reported on the $y$-axis of Figure 1 . 
Moreover, on the same graph, the three vertical dashed blue lines are marked for clusters having respectively $N=1695$, 4641, and 9855, corresponding to FeCo clusters with a diameter of around 3.7, 4.3, and $6.1 \mathrm{~nm}$ which are further experimentally studied. The three horizontal dash-dotted lines correspond to previous experimental distances $(\mathrm{FeFe}=2.78 \AA$, $\mathrm{CoCo}=2.74 \AA$, and $\mathrm{FeCo}=2.47 \AA$ ) obtained from EXAFS measurements on unselected FeCo clusters (with a median diameter of $\sim 3.2 \mathrm{~nm}$ and a large log-normal size dispersion of $\sim 0.45$ ) embedded in a carbon matrix after annealing. ${ }^{15}$ The different values obtained for homoatomic distances on unselected $\mathrm{FeCo}$ annealed clusters can qualitatively be related to element-specific relaxation effects, as already observed in chemically ordered CoPt nanoalloys. ${ }^{18}$ This effect is qualitatively reproduced by the DFT calculations. In addition to distances and magnetic moments computed for perfect B2 $\mathrm{FeCo}$ nanocrystallites, we have also considered extrinsic contributions related to the carbon environment: additional DFT calculations have been performed for a 65-atoms FeCo cluster, where one carbon atom is included in different surface sites. The main effects produced by a $\mathrm{C}$ impurity are to decrease the average spin polarization moment per atom and to produce local atomic distortions (see Figure S2). Moreover, we find that in calculations with two $\mathrm{C}$ impurities carbon atoms prefer to be separated from each other, favoring metalcarbon pair formation. When the number of added $\mathrm{C}$ atoms at the cluster surface is increased, the average spin polarization moment is all the more reduced as the magnetic moment per $\mathrm{Fe}$ or Co atom close to $\mathrm{C}$ atom is antiparallel coupled to the small magnetic moment $\left(\sim 0.2 \mu_{\mathrm{B}}\right)$ per $\mathrm{C}$ impurity.

\section{EXPERIMENTAL RESULTS AND DISCUSSION}

In Scheme 1, the mass-selected LECBD technique, described in detail elsewhere, ${ }^{19}$ is used to prepare benchmark size selected nanoparticles embedded in a carbon matrix. Pure or bimetallic clusters are produced in a laser vaporization source with the same stoichiometry as the target and then size selected in the gas phase by an electrostatic quadrupole deflector (used as a mass filter). ${ }^{20}$ Only cations are deflected, mass-selected, and deposited without coalescence on a substrate (amorphous carbon grid for TEM and Si wafer for other measurements) under ultrahigh-vacuum (UHV) conditions. Before transfer to air, all the samples are protected by a carbon layer coating, deposited using a homemade evaporator. $^{21}$ Both energy dispersive X-ray (EDX) and Rutherford backscattering (RBS) provide conclusive results concerning the equiatomic nature of the supported FeCo nanoparticles and show that there exists no sign of oxidation. ${ }^{10}$

From transmission electron microscopy (TEM) observations, the efficiency of the mass selection can be seen in the Supporting Information (Figure S3 and Table S2), where each cluster size histogram can be described by a Gaussian size distribution centered on a mean diameter $D_{\mathrm{m}}$ with a relative narrow size dispersion $\omega=\sigma_{D} / D_{\mathrm{m}}$ (where $\sigma_{D}$ is the standard deviation) of $\sim 0.1$ for both as-prepared pure and mixed clusters deposited on TEM grids. In the following, we present results obtained on samples of size-selected FeCo nanoparticles with three different mean diameters centered on 3.7, 4.3 , and $6.1 \mathrm{~nm}$, labeled $(\mathrm{FeCo})_{3 \mathrm{~nm}},(\mathrm{FeCo})_{4 \mathrm{~nm}}$, and $(\mathrm{FeCo})_{6 \mathrm{~nm}}$, respectively. A spherical shape is only observed for the as-prepared $(\mathrm{FeCo})_{3 \mathrm{~nm}}$ sample while ramified structures begin to form for the larger sizes $(\mathrm{FeCo})_{4 \mathrm{~nm}}$ and $(\mathrm{FeCo})_{6 \mathrm{~nm}}$ samples. As previously discussed, such particle morphology
Scheme 1. Overview of the Mass-Selected Cluster Generator Based on a Combined Nd:YAG Laser Vaporization/Inert He Gas Condensation Source, Associated with an Electrostatic Quadrupole Deviator and UHV Deposition Chamber; A Home-Made UHV Carbon Evaporator Is Used for in Situ Sample Preparation of Size-Selected Magnetic Nanoparticles Embedded in a Carbon Matrix (Bottom Right)

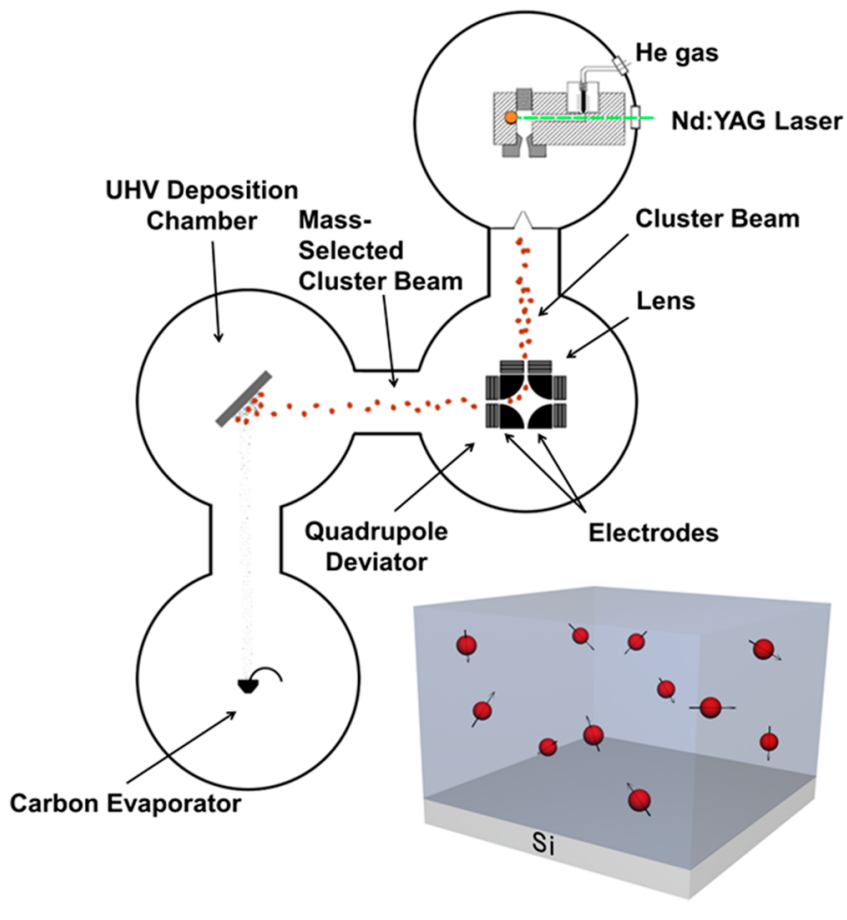

changes depend on the growth kinetics of metal clusters in the gas phase which are governed by external parameters (laser power, carrier gas pressure, etc.). ${ }^{22}$

To reach the equilibrium phase, as-prepared FeCo samples have been annealed under UHV conditions at a temperature of $500{ }^{\circ} \mathrm{C}$ for $2 \mathrm{~h}$ and reimaged for conventional size histograms. Annealing systematically induces a morphology change to more energetically favorable spherical nanoparticles with no coalescence between NPs, as shown in the TEM images and histograms obtained before and after annealing on massselected $(\mathrm{FeCo})_{4 \mathrm{~nm}}$ and $(\mathrm{FeCo})_{6 \mathrm{~nm}}$ samples (Figure 2$)$. The corresponding mean diameters and dispersions are reported in Table 1 where the larger the initial diameter, the greater the decrease in diameter after annealing (up to $13 \%$ for the $(\mathrm{FeCo})_{6 \mathrm{~nm}}$ sample). This apparent size reduction upon annealing may be explained by the fact that the diameter, estimated from TEM images using the projected area of a nanoparticle (ellipsoidal model), can be inaccurate and overestimated for nonspherical morphologies.

As for unselected clusters, ${ }^{15}$ conventional high-resolution TEM (HRTEM) observations do not permit us to distinguish a chemically ordered B2 structure from random bodycentered-cubic A2 (W-type) structure (see Figure S4). With the development of spherical aberration correction technology for electron microscopy, sophisticated atomic-scale chemical mapping in the scanning transmission electron microscopy (STEM) geometry utilizing combination of energy-dispersive X-ray spectra (STEM-EDS) and electron-energy-loss spectra (STEM-EELS) signal could have been used to try to 
a)

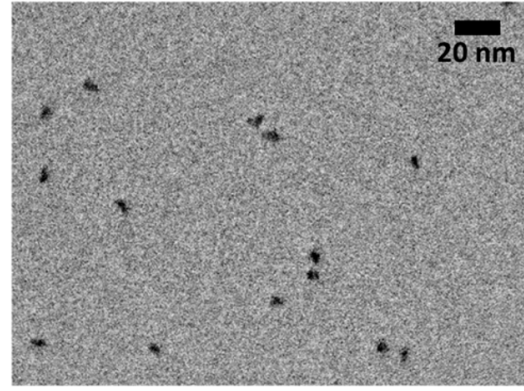

b)

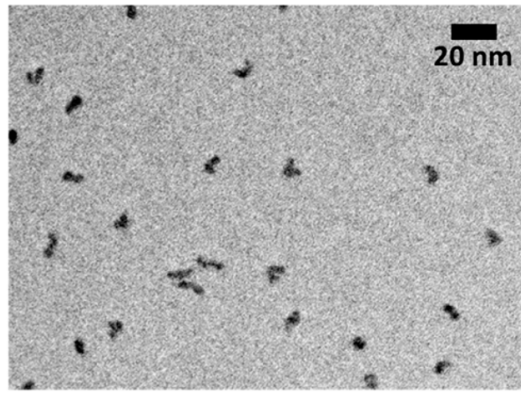

c)
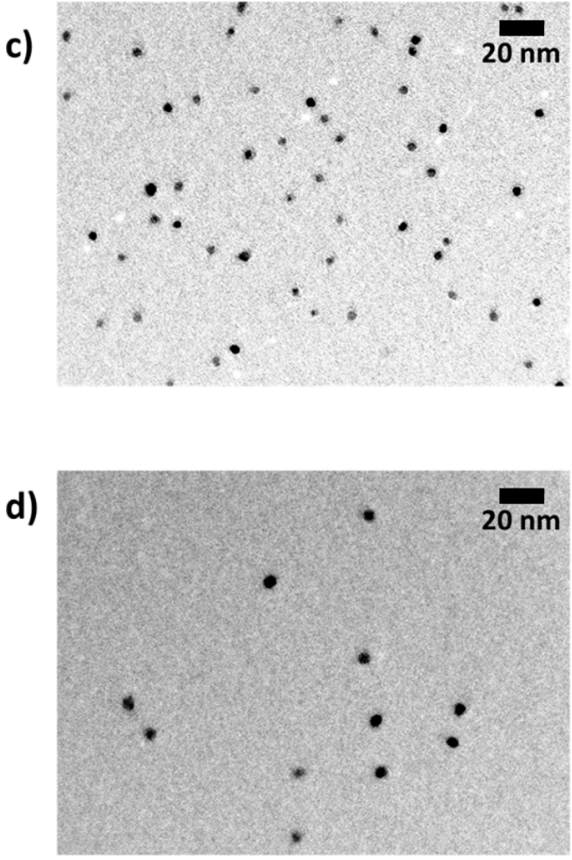
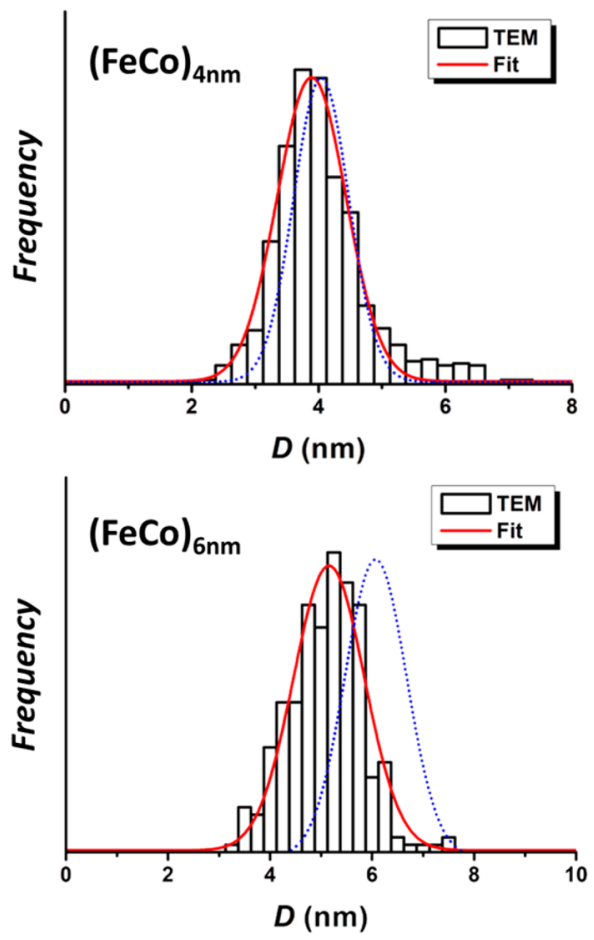

Figure 2. TEM images for as-prepared mass-selected FeCo nanoparticles (a, b) and upon annealing at $500{ }^{\circ} \mathrm{C}$ for $2 \mathrm{~h}$ in $\mathrm{UHV}$ (c, d) for the $(\mathrm{FeCo})_{4 \mathrm{~nm}}(\mathrm{top})$ and $(\mathrm{FeCo})_{6 \mathrm{~nm}}$ (bottom) sample with corresponding size histogram before (including Gaussian fit in the blue dotted line) and after annealing (including Gaussian fit in the red solid line).

Table 1. Mean Diameter (Ellipsoidal Model) and Dispersion Obtained from TEM Histogram Simulations on As-Prepared and Annealed $(\mathrm{FeCo})_{4 \mathrm{~nm}}$ and $(\mathrm{FeCo})_{6 \mathrm{~nm}}$ Samples

\begin{tabular}{cccccc} 
& \multicolumn{2}{c}{ as-prepared } & & \multicolumn{2}{c}{ annealed } \\
\cline { 2 - 3 } \cline { 5 - 6 } FeCo samples & $D_{m}(\mathrm{~nm}) \pm 0.2$ & $\omega \pm 0.03$ & & $D_{m}(\mathrm{~nm}) \pm 0.2$ & $\omega \pm 0.03$ \\
$(\mathrm{FeCo})_{4 \mathrm{~nm}}$ & 4.3 & 0.12 & & 3.9 & 0.14 \\
$(\mathrm{FeCo})_{6 \mathrm{~nm}}$ & 6.1 & 0.13 & & 5.3 & 0.13
\end{tabular}

discriminate the crystal lattice and chemical structure on a number of intermetallic $\mathrm{FeCo} \mathrm{NPs}^{23}$

Our alternative strategy has been to use statistical techniques based on the chemical selectivity of X-rays absorption (XAS) performed on thick samples made up of a $2 \mathrm{D}$ configuration of alternating layers of mass-selected clusters and amorphous carbon matrix with a total of 28 layers of clusters (corresponding to $\sim 10^{14}$ clusters per sample) deposited on the Si substrate, as illustrated in Scheme 1. We have performed XAS experiments at both $\mathrm{Fe}$ and $\mathrm{Co} \mathrm{K}$-edges because EXAFS measurements provide quantitative information about the local structure near the probed atom and on the nature of its neighbors. To better separate the influence of annealing, alloying, and size effects, we systematically investigate identical pairs of samples: one as-prepared and one annealed $\mathrm{FeCo}$ nanoparticle sample. In the following, we discuss the thermal evolution in the Fourier transform (FT) of the EXAFS oscillations at each edge, assimilated to the nearest-neighbor (NN) radial distribution in specific atomic environments. For reference, we also study pure $4 \mathrm{~nm}$ size-selected $\mathrm{Fe}$ and Co clusters presented in the Supporting Information. In Figure S5, one can see that upon annealing the intensity of the first NN peak (located at $2 \AA$ ) in the Co nanoparticles increases due to a crystal coordination enhancement. The carbide shoulder present in the first NN peak for both as-prepared samples almost disappears in annealed Co clusters, while $\mathrm{Fe}-\mathrm{C}$ bonds remain predominant in annealed Fe nanoparticles. This allows us to define two trends: one for iron particles, where annealing is unable to get rid of carbon insertion, and one for cobalt particles, where annealing expels the carbon atoms from the particles back to the matrix, as already observed from magnetic measurements. ${ }^{25}$

For the as-prepared bimetallic FeCo clusters, the three nanoparticle sizes exhibit qualitatively the same XAS signals at both edges (see Figures S6 and S7), and the EXAFS simulations are very similar to the local crystallographic parameters obtained for the unselected $\mathrm{FeCo}$ sample. ${ }^{15}$

On the contrary, after annealing, while a disordered structure persists for smaller FeCo particles (see Figures S8 and S9), a strong structuration in the radial distribution is observed on the larger $(\mathrm{FeCo})_{6 \mathrm{~nm}}$ sample (see Figure $3 \mathrm{~b}, \mathrm{e}$ ) which is completely different from that of the $(\mathrm{FeCo})_{4 \mathrm{~nm}}$ sample (see Figure 3a,d).

In the case of the annealed $(\mathrm{FeCo})_{6 \mathrm{~nm}}$ sample, a shift in the first NN peak with a large intensity increase (it is more than doubled) is observed as well as three new peaks resulting from the next NN scattering up to $6 \AA$. Noticeably, the pseudo radial pair distribution at both edges is very similar to the one of a metallic Fe bcc foil (see Figure S10). Because a bcc pure Co structure cannot be formed, this indicates that Co necessarily has Fe neighboring atoms. In addition, looking at the usual carbide prepeak $(\sim 1.5 \AA)$, this shoulder is strongly diminished at the iron edge and almost disappears at the cobalt edge. But the best evidence of higher carbide stability for the iron environment can be found in the broader first $\mathrm{NN}$ peak at the $\mathrm{Fe}$ K-edge compared to the one at the Co K-edge. This broadening is probably related to local atomic distortions 


\section{Fe K-Edge}

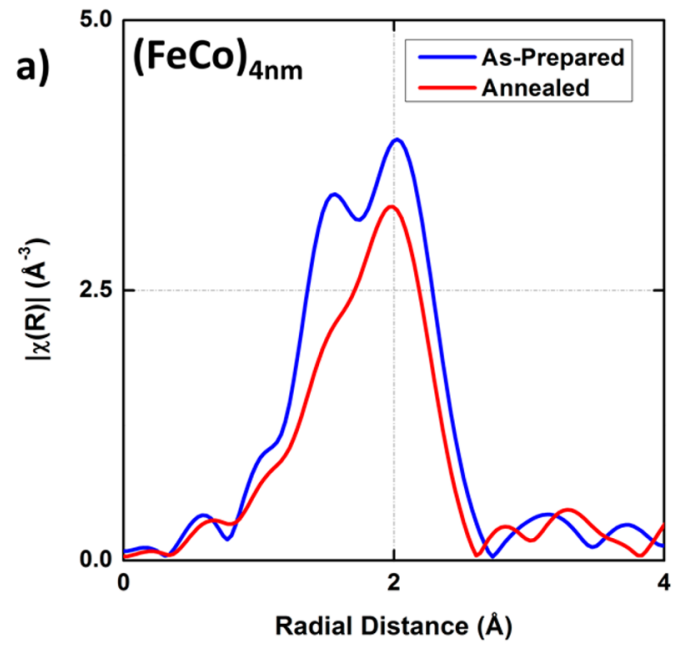

b)

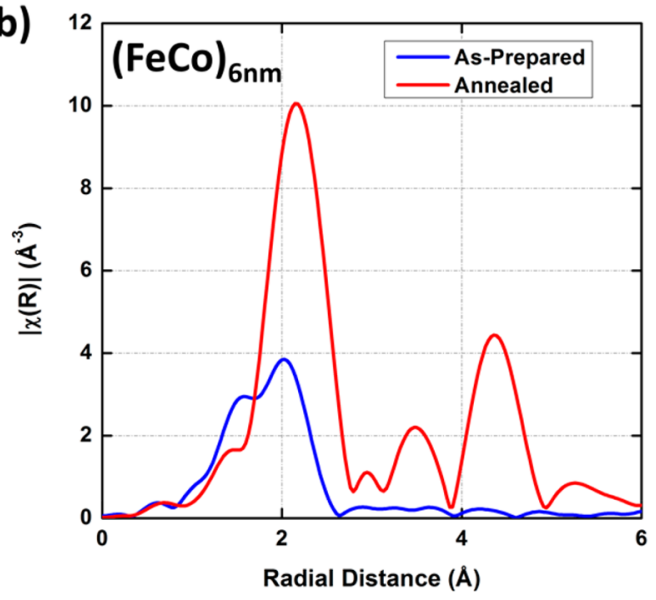

c)

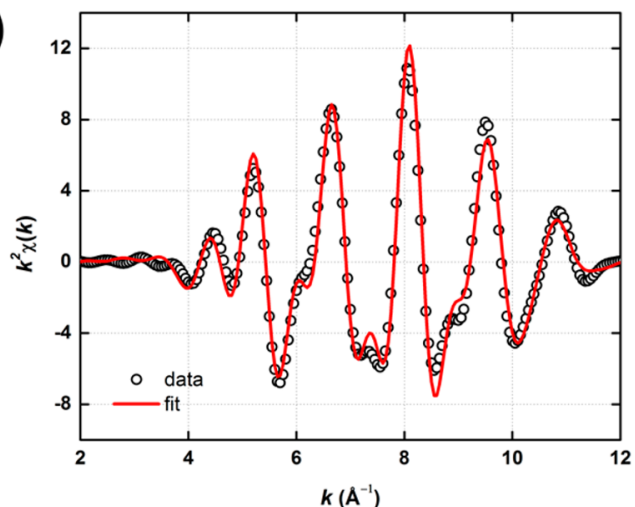

\section{Co K-Edge}

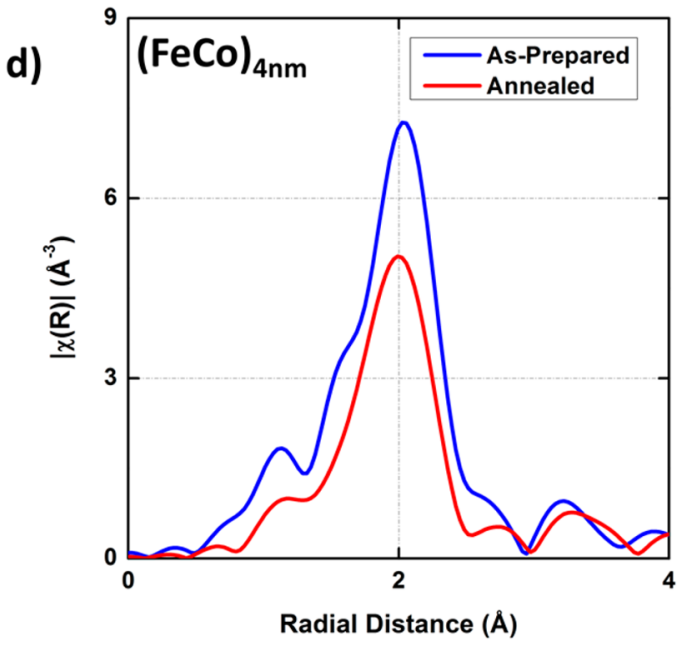

e)

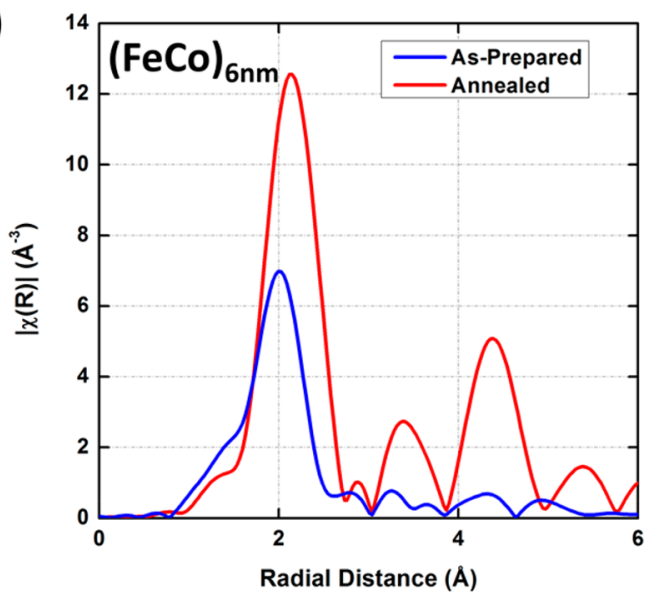

f)

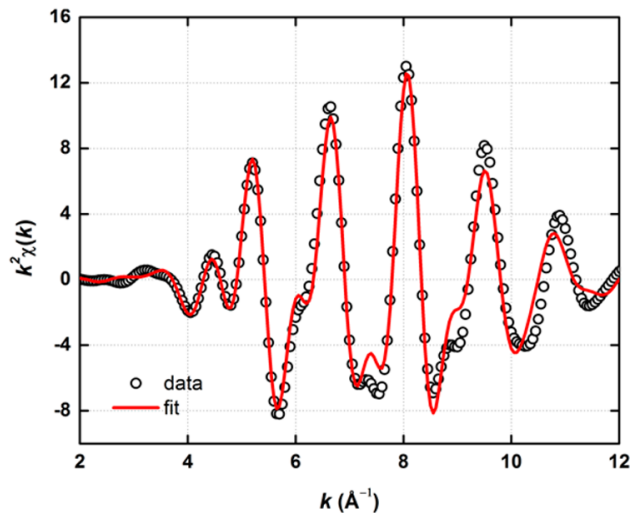

Figure 3. Thermal evolution of the NN radial distribution evolution at Fe K-edge (left) and Co K-edge (right) for the (FeCo) $4 \mathrm{~nm}(\mathrm{a}, \mathrm{d})$ and $(\mathrm{FeCo})_{6 \mathrm{~nm}}(\mathrm{~b}, \mathrm{e})$ samples. EXAFS oscillations and corresponding best fits (in red solid line) for the annealed (FeCo) ${ }_{6 \mathrm{~nm}}$ sample at the Fe K-edge (c) and Co (f) K-edge.

induced by carbon impurities, as exemplified by DFT calculations.

A quantitative EXAFS analysis is performed by fitting the $k^{2}$ weighted function $\chi(k)$ to the standard EXAFS formula using the IFEFFIT software package and a perfect B2 phase. $^{24}$ The EXAFS oscillations and corresponding best fits at the $\mathrm{Fe}$ and Co K-edge for the annealed $(\mathrm{FeCo})_{6 \mathrm{~nm}}$ sample are presented in Figure $3 c$,f. The results of best fits are tabulated in Table 2. Notice that in the bulk B2 phase there are eight homoatomic NN (in the centers of the neighbored cubes) and six heteroatomic next NN (located at the neighbored vertices of the lattice). For this $(\mathrm{FeCo})_{6 \mathrm{~nm}}$ sample, we obtained reduced 5.1 (4.9) NN and 3.8 (3.7) next NN at the Fe edge (Co edge) which can be related to broken metallic bonds at the NP surface (see Table 2). Moreover, setting the NN ratio for each species to the $8 / 6$ value of the bulk $\mathrm{B} 2$ phase leads to a very good agreement. The homoatomic distances $\left(d_{\mathrm{Fe}-\mathrm{Fe}}=d_{\mathrm{Co}-\mathrm{Co}}\right)$ correspond to a single distance of $\sim 2.80 \AA$, and the heteroatomic distance $\left(d_{\mathrm{Co}-\mathrm{Fe}}=d_{\mathrm{Fe}-\mathrm{Co}}\right)$ is equal to $2.46 \AA$ at both edges (the ratio between these distances is $\sqrt{ } 3 / 2 \cong 0.866$ 
Table 2. Coordination NN Numbers, Debye Waller Value $\left(\sigma^{2}\right.$ in $\left.\AA^{2}\right)$, and Average Interatomic Distances ( $R$ in $\AA$ with a 0.01 A Error Bar) Obtained from the Best Fits of the EXAFS Oscillations for the Annealed $(\mathrm{FeCo})_{6 \mathrm{~nm}}$ Sample at the Fe and Co K-Edge

$\begin{array}{ccccc}\text { Fe K-edge } & \text { path } & \text { NN number } & \sigma^{2}\left(\AA^{2}\right) & R(\AA) \\ & \text { Fe-Co } & 5.1 & 0.0089 & 2.46 \pm 0.02 \\ & \text { Fe-Fe } & 3.8 & 0.0104 & 2.80 \pm 0.02 \\ & \text { Fe-C } & 0.6 & 0.0273 & 2.02 \pm 0.02 \\ \text { Co K-edge } & \text { path } & \text { NN number } & \sigma^{2}\left(\AA^{2}\right) & R(\AA) \\ & \text { Co-Fe } & 4.9 & 0.0089 & 2.46 \pm 0.02 \\ & \text { Co-Co } & 3.7 & 0.0098 & 2.81 \pm 0.02\end{array}$

for the B2 phase). All these experimental distances are reported as stars in Figure 1. Even with a Debye-Waller parameter $\sigma^{2}$ relatively large $\left(\sim 10^{-2} \AA^{2}\right)$ for intermetallic NN, carbon neighbors are needed to be introduced to improve the quality of the EXAFS fit but only for the iron edge. This suggests that $\mathrm{C}$ atoms mostly occupy interstitial sites near the $\mathrm{Fe}$ atoms and that the surface is made of $\mathrm{Fe}$ atoms, thus enabling the formation of carbon-iron bonds at the cluster shell. Nevertheless, EXAFS measurements are in favor of a well-ordered B2 bimetallic phase at the cluster core.

As we have previously shown that interface intermixingeven through one monolayer-can drastically change the magnetic properties, ${ }^{25}$ in the next section, we present both $\mathrm{X}$ ray magnetic circular dichroism (XMCD) and (SQUID) magnetometry measurements performed on the same samples.

First, we determine the spin $\left(\mu_{s}\right)$ and orbital $\left(\mu_{L}\right)$ moments from XMCD measurement on pure and bimetallic nanoalloys samples at both $\mathrm{Fe}$ and Co L-edges. ${ }^{26}$ For pure samples, we clearly identify two different XMCD thermal evolutions. For the as-prepared pure $\mathrm{Fe}_{(3 \mathrm{~nm})}$ sample, we obtain a small total magnetic moment $\left(\mu=\mu_{s}+\mu_{L}\right)$ (only equal to $60 \%$ of the $\mathrm{Fe}$ bulk $\mu$ value), which decreases again by $30 \%$ after thermal treatment. According to ab initio calculations, such a reduction in $\mu$ can be explained by carbide enrichment due to the annealing process on the iron environment. For the pure asprepared $\mathrm{Co}_{(3 \mathrm{~nm})}$ sample, we obtain an equivalently small $\mu$ value (equal to $60 \%$ of the Co-bulk $\mu$ value) which almost doubled upon annealing. Such thermal $\mu$ enhancement per Co atom can be explained by the annealing carbon demixing process in the cobalt environment. By comparison of the
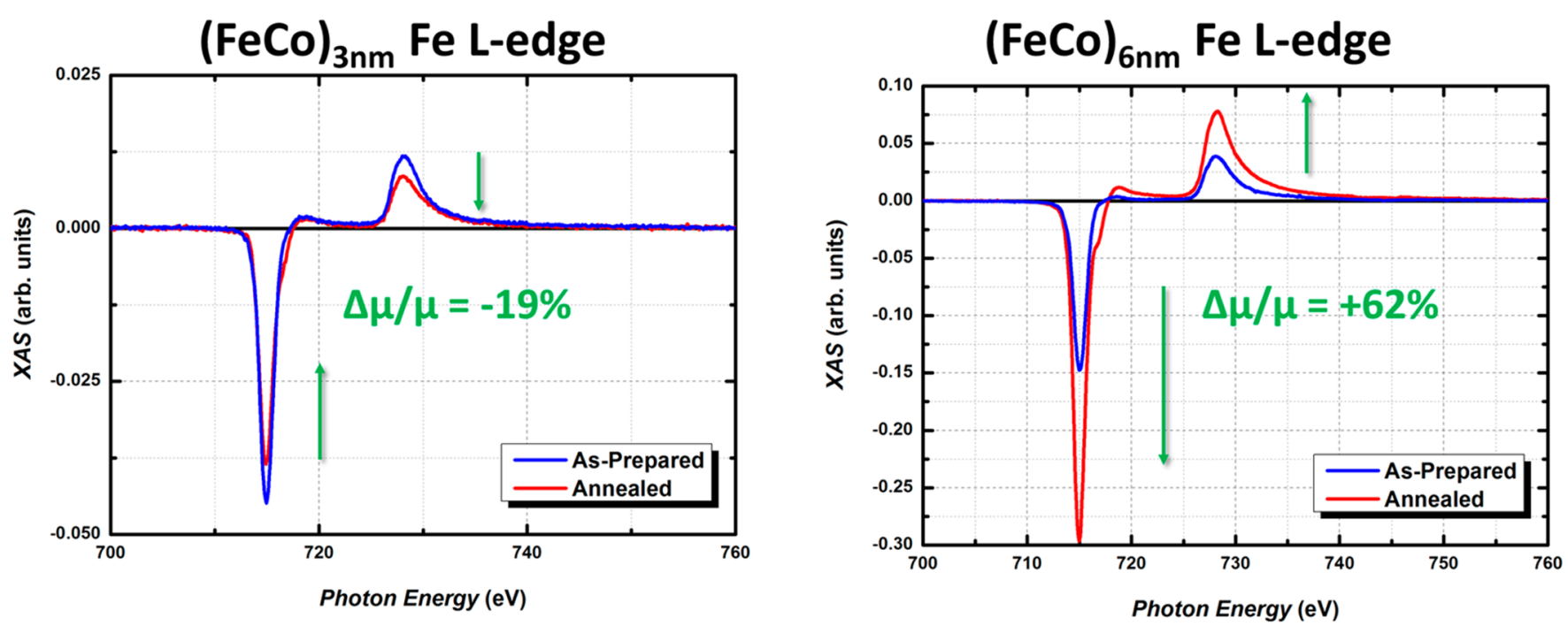

$(\mathrm{FeCO})_{3 \mathrm{~nm}} \mathrm{Co}$ L-edge

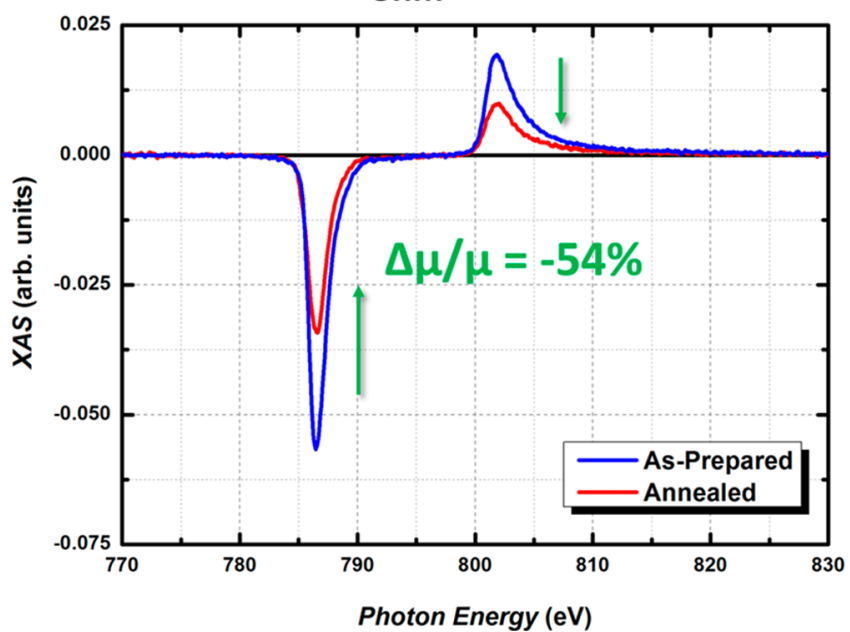

$(\mathrm{FeCo})_{6 \mathrm{~nm}}$ Co L-edge

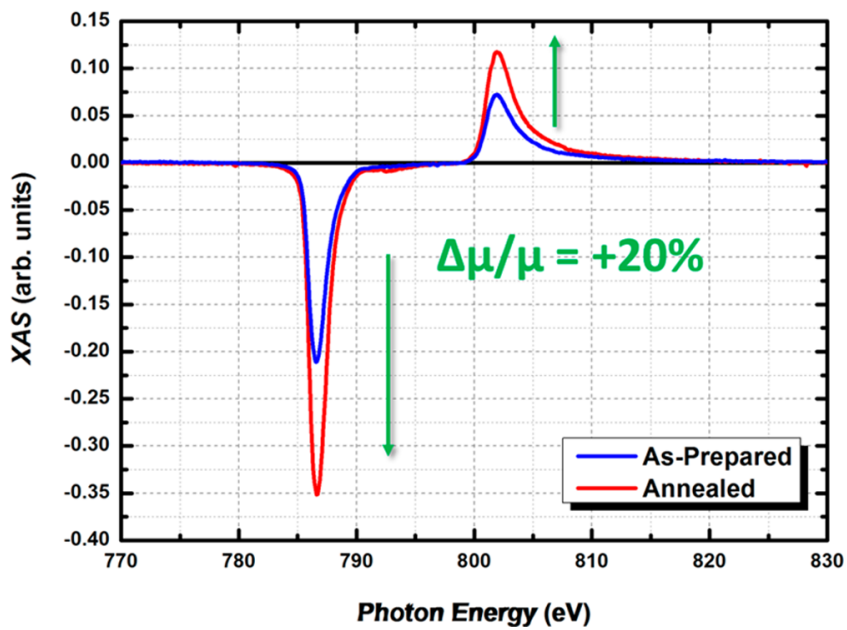

Figure 4. XMCD thermal evolution at both $\mathrm{Fe}$ (top) and Co (bottom) L-edges for $(\mathrm{FeCo})_{3 \mathrm{~nm}}$ (left) and (FeCo) $6 \mathrm{~nm}$ (right) samples before (in blue) and after annealing (in red). In green is noted the corresponding $\Delta \mu / \mu$ relative thermal variation of total moment. 


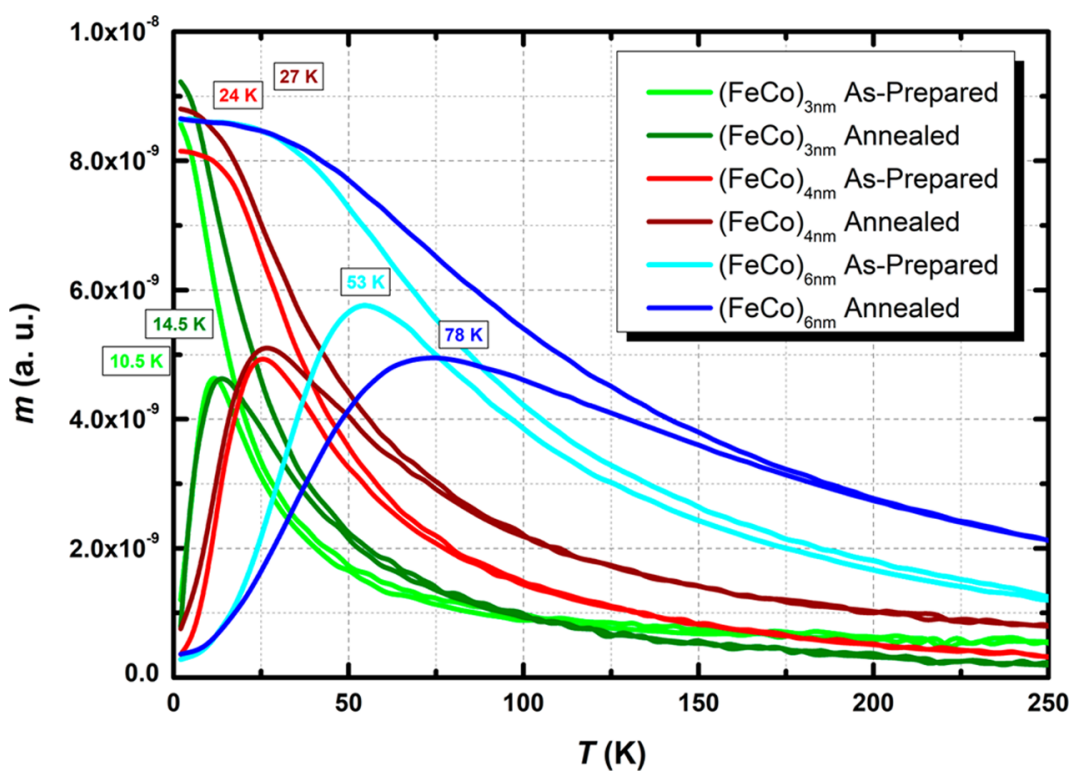

Figure 5. ZFC/FC susceptibility curves obtained before (in light) and after annealing (in dark) of (FeCo) $3 \mathrm{~nm}$ (in green), $(\mathrm{FeCo})_{4 \mathrm{~nm}}($ in blue), and $(\mathrm{FeCo})_{6 \mathrm{~nm}}($ in red $)$ samples.

$\mathrm{XMCD}$ thermal evolution for $(\mathrm{FeCo})_{3 \mathrm{~nm}}$ and $(\mathrm{FeCo})_{6 \mathrm{~nm}}$ samples, Figure 4 shows that the total magnetic moment $(\mu$ $\left.=\mu_{s}+\mu_{L}\right)$ is reduced (enhanced) for annealed small (large) FeCo nanoparticles embedded in a carbon matrix. Thus, the $(\mathrm{FeCo})_{3 \mathrm{~nm}}$ sample follows the Fe magnetic trend even at the Co edge while the $(\mathrm{FeCo})_{6 \mathrm{~nm}}$ samples follows the Co magnetic trend with enhanced moments close to the that of the bulk at the Co edge after annealing and halved at the Fe edge. The $(\mathrm{FeCo})_{4 \mathrm{~nm}}$ sample corresponds to an intermediate trend where XMCD signal shows an enhancement at the Co edge, while the Fe edge remains almost unchanged upon annealing.

Then by studying the zero-field cooled (ZFC)/field cooled (FC) susceptibility curves as a function of temperature, we observe that the maximum of the ZFC susceptibility curves (indicated as $T_{\max }$ on the Figure 5) increases with the magnetic size of the cluster. This can be explained by the fact that above $T_{\max }$ the superparamagnetic state occurs when the effective magnetic anisotropy energy (MAE), proportional to the anisotropy constant and to the volume of nanomagnets $\Delta E$ $=K_{1} V$, is counterbalanced by the thermal energy $k_{\mathrm{B}} T$. We also observe a significant $T_{\max }$ enhancement upon annealing for the largest FeCo clusters size. By adjusting SQUID magnetic measurements from triple fit method ${ }^{27}$ and the total moment values determined from XMCD, we obtain the corresponding parameters presented in Table 3 . We observe a significant $K_{1}$ increase of $37 \%$ for the annealed $(\mathrm{FeCo})_{6 \mathrm{~nm}}$ sample with almost the same magnetic diameter as in TEM. Once more,

Table 3. Maxima of the ZFC $\left(T_{\max }\right)$, Coercive Field $\left(\mu_{0} H_{\mathrm{C}}\right)$, and the Deduced Parameters from Triple Fit Adjustment of the SQUID Measurements for the As-Prepared $(\mathrm{FeCo})_{6 \mathrm{~nm}}$ Sample and after Annealing

\begin{tabular}{|c|c|c|c|c|c|}
\hline & $\begin{array}{l}T_{\max } \\
(\mathrm{K})\end{array}$ & $\begin{array}{l}\mu_{0} H_{\mathrm{C}} \\
(\mathrm{mT})\end{array}$ & $\underset{\omega_{\mathrm{mag}}}{D_{\mathrm{mag}}(\mathrm{nm})}$ & $M_{s}\left(\mathrm{kA} \mathrm{m}^{-1}\right)$ & $\frac{K_{1}\left(\mathrm{~kJ} \mathrm{~m}^{-3}\right)}{\omega_{K_{1}}}$ \\
\hline $\begin{array}{l}\text { as- } \\
\text { prepared }\end{array}$ & 53 & 101 & $\begin{array}{l}6.2 \pm 0.2 \\
0.08 \pm 0.02\end{array}$ & $1000 \pm 100$ & $\begin{array}{l}120 \pm 10 \\
0.34 \pm 0.05\end{array}$ \\
\hline annealed & 78 & 121 & $\begin{array}{l}6.1 \pm 0.2 \\
0.12 \pm 0.02\end{array}$ & $1200 \pm 100$ & $\begin{array}{l}165 \pm 10 \\
0.40 \pm 0.05\end{array}$ \\
\hline
\end{tabular}

this MAE evolution is probably due to the thermal structural evolution from a disordered phase to a main B2 phase obtained for the largest FeCo nanoparticles embedded in a carbon matrix.

\section{CONCLUSION}

On the basis of direct correlation between structural and magnetic properties, we evidence size-dependent thermal tendencies for equiatomic $\mathrm{FeCo}$ nanoparticles in a carbon environment. First, we show that at small $3 \mathrm{~nm}$ size the FeCo clusters behave like pure $\mathrm{Fe}$ clusters where annealing increases carbon diffusion into the NPs, thus causing a reduction of the average magnetic moment per atom. While at the larger $6 \mathrm{~nm}$ size the FeCo clusters behave like Co clusters where annealing helps demixing the carbon atoms from the clusters, thus leading to an increased average magnetic moment per atom. At the intermediate $4 \mathrm{~nm}$ FeCo cluster size, there is a progressive change between these two chemical reactivity tendencies. Thus, it appears that our results should provide a better basic understanding of the true influence of size and alloy effects on the FeCo catalytic performance. For example, one can assume that in the F-T process an iron-rich surface layer prevents the cobalt oxidization in FeCo catalysts which exhibit a higher carbon monoxide conversion and hydrocarbon selectivity than in pure cobalt catalysts under similar process conditions. ${ }^{3}$ This could also explain the synergistic effect in active-active bimetallic FeCo catalysts where the presence of Co is suspected to facilitate $\mathrm{Fe}$ reduction for the synthesis of nanotube forests more efficiently than with pure catalyst. ${ }^{2}$

As an overall conclusion, we show for the first time that carbon solubility effects are size dependent in FeCo nanoparticles and that above a critical $5 \mathrm{~nm}$ diameter the iron carbide interface promotes a thermally activated transition to the chemically ordered B2 phase in the alloyed FeCo core, which is especially interesting for efficient high-frequency magnetic materials. ${ }^{14}$ Complementary thermodynamic models could be interesting to calculate to establish a precise sizedependent nanocrystalline $\mathrm{FeCo}-\mathrm{C}$ phase diagram as previously done for $\alpha$-phase $\mathrm{Fe}-\mathrm{C}$ nanoalloys. ${ }^{28}$ To go a step 
further, it would be essential to test the performance in real conditions of such naked mass-selected FeCo NPs as more active, more selective, more stable, and less expensive FischerTropsch catalysts. Investigation of evolution of their physicochemical properties under a reactant gas mixture of $\mathrm{CO}$ and $\mathrm{H}_{2}$ could be performed at different steps of redox reactions thanks to advanced experimental characterization tools available at synchrotron radiation facilities. ${ }^{29}$

\section{ASSOCIATED CONTENT}

\section{S Supporting Information}

The Supporting Information is available free of charge on the ACS Publications website at DOI: 10.1021/acsanm.9b00313.

Supplementary details, figures, and tables on theoretical DFT calculations, geometrical descriptions of B2-FeCo clusters and carbon atom surface inclusion (1), on experimental TEM and HRTEM observations (2); EXAFS measurements and simulations (3) obtained on mass-selected as-prepared and annealed pure and bimetallic FeCo clusters (PDF)

\section{AUTHOR INFORMATION}

\section{Corresponding Author}

*E-mail Veronique.Dupuis@univ-lyon1.fr.

\section{ORCID}

Veronique Dupuis: 0000-0001-6225-6608

Juan Martín Montejano-Carrizales: 0000-0002-6677-0783

Florent Tournus: 0000-0001-8149-7597

Faustino Aguilera-Granja: 0000-0002-8925-3180

\section{Notes}

The authors declare no competing financial interest.

\section{ACKNOWLEDGMENTS}

The authors are very grateful to $\mathrm{C}$. Albin and O. Boisron from the PLYRA iLM platforms, O. Proux on the French BM30bFAME beamlines at ESRF, and P. Ohresser on the DEIMOSFrench national SOLEIL synchrotron for their technical support and fruitful discussions. Both J.M.M.C. and F.A.G. acknowledge Jose Limón (UASLP) and Diego Lasa (DIPC) for computational support and suggestions. All the SQUID measurements were performed at the CML platform. Support is acknowledged from the International Research Network (IRN)/Groupement de Recherche International (GDRI) on Nanoalloys.

\section{REFERENCES}

(1) Løvik, A. N.; Hagelüken, C.; Wäger, P. Improving Supply Security of Critical Metals: Current Developments and Research in the EU. Sustainable Materials and Technologies 2018, 15, 9-18.

(2) Hardeman, D.; Esconjauregui, S.; Cartwright, R.; Bhardwaj, S.; D’Arsié, L.; Oakes, D.; Clark, J.; Cepek, C.; Ducati, C.; Robertson, J. The Synergistic Effect in the Fe-Co Bimetallic Catalyst System for the Growth of Carbon Nanotube Forests. J. Appl. Phys. 2015, 117, No. 044308.

(3) Calderone, V. R.; Shiju, N. R.; Ferré, D. C.; Rothenberg, G. Bimetallic Catalysts for the Fischer-Tropsch Reaction. Green Chem. 2011, 13, 1950-1959.

(4) Van Santen, R. A. Complementary Structure Sensitive and Insensitive Catalytic Relationships. Acc. Chem. Res. 2009, 42, 57-66.

(5) Ferrando, R.; Jellinek, J.; Johnston, R. L. Nanoalloys: from Theory to Applications of Alloy Clusters and Nanoparticles. Chem. Rev. 2008, 108, 845-910.
(6) Bozorth, R. M. Ferromagnetism; D. Van Nostrand Co.: New York, 1951; Chapter XII.

(7) Ohnuma, I.; Enoki, H.; Ikeda, O.; Kainuma, R.; Ohtani, H.; Sundman, B.; Ishida, K. Phase Equilibria in the Fe-Co Binary System. Acta Mater. 2002, 50, 379-393.

(8) Fish, G. E. Soft Magnetic Materials. Proc. IEEE 1990, 78, 947972.

(9) Aguilera-Granja, F.; Vega, A. Stability, Magnetic Behavior, and Chemical Order of $\left(\mathrm{Co}_{\mathrm{x}} \mathrm{Fe}_{1-\mathrm{x}}\right)_{\mathrm{N}}(\mathrm{N}=5,13)$ Nanoalloys. Phys. Rev. B: Condens. Matter Mater. Phys. 2009, 79, 144423.

(10) Khadra, G.; Tamion, A.; Tournus, F.; Canut, B.; Dupuis, V. Anisotropy in FeCo Nanoparticles, a First Step. Solid State Phenom. 2015, 233-234, 550-553.

(11) Delczeg-Czirjak, E. K.; Pereiro, M.; Bergqvist, L.; Kvashnin, Y. O.; Di Marco, G.; Li, I.; Vitos, L.; Eriksson, O. Stabilization of the Tetragonal Distortion of $\mathrm{Fe}_{\mathrm{x}} \mathrm{Co}_{1-\mathrm{x}}$ Alloys by C Impurities: A Potential New Permanent Magnet. Phys. Rev. B: Condens. Matter Mater. Phys. 2014, 90, 144403.

(12) Dupuis, V.; Robert, A.; Hillion, A.; Khadra, G.; Blanc, N.; Le Roy, D.; Tournus, F.; Albin, C.; Boisron, O.; Tamion, A. Cubic Chemically Ordered FeRh and FeCo Nanomagnets Prepared by Mass-Selected Low-Energy Cluster-Beam Deposition: a Comparative Study. Beilstein J. Nanotechnol. 2016, 7, 1850-1860.

(13) Liu, Y.; Luo, J.; Shin, Y.; Moldovan, S.; Ersen, O.; Hébraud, A.; Schlatter, G.; Pham-Huu, C.; Meny, C. Sampling the Structure and Chemical Order in Assemblies of Ferromagnetic Nanoparticles by Nuclear Magnetic Resonance. Nat. Commun. 2016, 7, 11532.

(14) Garnero, C.; Lepesant, M.; Garcia-Marcelot, C.; Shin, Y.; Meny, C.; Farger, P.; Warot-Fonrose, B.; Arenal, R.; Viau, G.; Soulantica, K.; Fau, P.; Poveda, P.; Lacroix, L.-M.; Chaudret, B. Chemical Ordering in Bimetallic FeCo Nanoparticles: From a Direct Chemical Synthesis to Application as Efficient High-Frequency Magnetic Material. Nano Lett. 2019, 19, 1379-1386.

(15) Khadra, G.; Tamion, A.; Tournus, F.; Boisron, O.; Albin, C.; Dupuis, V. Structure and Magnetic Properties of FeCo Clusters: Carbon Environment and Annealing Effects. J. Phys. Chem. C 2017, 121, 10713-10718.

(16) Magnin, Y.; Zappelli, A.; Amara, H.; Ducastelle, F.; Bichara, C. Size Dependent Phase Diagrams of Nickel-Carbon Nanoparticles. Phys. Rev. Lett. 2015, 115, 205502-5.

(17) Soler, J. M.; Artacho, E.; Gale, J. D.; Garcia, A.; Junquera, J.; Ordejon, P.; Sanchez-Portal, D. The SIESTA Method for ab initio Order-N Materials Simulation. J. Phys.: Condens. Matter 2002, 14, $2745-2779$.

(18) Blanc, N.; Diaz-Sanchez, L. E.; Ramos, A. Y.; Tournus, F.; Tolentino, H. C. N.; De Santis, M.; Proux, O.; Tamion, A.; TuaillonCombes, J.; Bardotti, L.; Boisron, O.; Pastor, G. M.; Dupuis, V. Element-Specific Quantitative Determination of the Local Atomic Order in CoPt Alloy Nanoparticles: Experiment and Theory. Phys. Rev. B: Condens. Matter Mater. Phys. 2013, 87, 155412.

(19) Perez, A.; Dupuis, V.; Tuaillon-Combes, J.; Bardotti, L.; Prevel, B.; Bernstein, E.; Melinon, P.; Favre, L.; Hannour, A.; Jamet, M. Functionalized Cluster-Assembled Magnetic Nanostructures for Applications to High Integration-Density Devices. Adv. Eng. Mater. 2005, 7, 475 .

(20) Tournus, F.; Blanc, N.; Tamion, A.; Hillenkamp, M.; Dupuis, V. Synthesis and Magnetic Properties of Size-Selected CoPt Nanoparticles. J. Magn. Magn. Mater. 2011, 323, 1868.

(21) Patent Number WO/2014/191688.

(22) Alayan, R.; Arnaud, L.; Broyer, M.; Cottancin, E.; Lerme, J.; Vialle, J. L.; Pellarin, M. Morphology and Growth of Metal Clusters in the Gas Phase: A Transition from Spherical to Ramified Structures. Phys. Rev. B: Condens. Matter Mater. Phys. 2006, 73, 125444.

(23) Lu, P.; Zhou, L.; Kramer, M. J.; Smith, D. J. Atomic-Scale Chemical Imaging and Quantification of Metallic Alloy Structures by Energy-Dispersive X-Ray Spectroscopy. Sci. Rep. 2015, 4, 3945.

(24) Ravel, B.; Newville, M. ATHENA, ARTEMIS, HEPHAESTUS: Data Analysis for X-Ray Absorption Spectroscopy using IFEFFIT. J. Synchrotron Radiat. 2005, 12, 537. 
(25) Tamion, A.; Hillenkamp, M.; Hillion, A.; Tournus, F.; TuaillonCombes, J.; Boisron, O.; Zafeiratos, S.; Dupuis, V. Demixing in Cobalt Clusters Embedded in a Carbon Matrix Evidenced by Magnetic Measurements. J. Appl. Phys. 2011, 110, No. 063904.

(26) Khadra, G. Magnetic and Structural Properties of Size-Selected FeCo Nanoparticle Assemblies. PhD Thesis, iLM Lyon, 2015; https://tel.archives-ouvertes.fr/tel-01262653.

(27) Tamion, A.; Hillenkamp, M.; Tournus, F.; Bonet, E.; Dupuis, V. Accurate Determination of the Magnetic Anisotropy in ClusterAssembled Nanostructures. Appl. Phys. Lett. 2009, 95, No. 062503.

(28) Kirchner, A.; Kieback, B. Solubility of Carbon in Nanocrystalline $\alpha$-Iron. J. Nanomater. 2012, 2012, 1.

(29) Lee, S.; Lee, B.; Seifert, S.; Winans, R. E.; Vajda, S. FischerTropsch Synthesis at a Low Pressure on Subnanometer Cobalt Oxide Clusters: The Effect of Cluster Size and Support on Activity and Selectivity. J. Phys. Chem. C 2015, 119 (20), 11210-11216. 